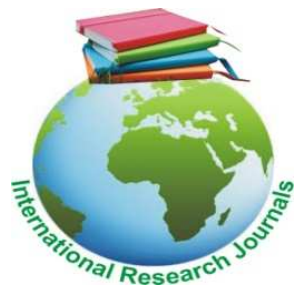

Journal of Medicine and Medical Sciences Vol. 8(2) pp. 014-019, April 2017

Available online http://www.interesjournals.org/JMMS

DOI: http:/dx.doi.org/10.14303/jmms.2017.018

Copyright (C) 2017 International Research Journals

Full Length Research Paper

\title{
Human avian influenza virus serotypes in a tertiary hospital in Nigeria
}

\author{
*Jacob A Dunga1, Alkali Mohammed', Nura H Alkali', Abubakar A Gombe ${ }^{2}$, Yusuf B Jibrin', \\ Sabiyu A Gwalabe ${ }^{1}$, Jafiada J Musa ${ }^{1}$, Mustapha S Umar ${ }^{1}$, Suleiman Y Yusuf ${ }^{3}$, Aishatu Bello ${ }^{1}$, \\ Binta Lassan ${ }^{1}$
}

\author{
${ }^{1}$ Abubakar Tafawa Balewa Teaching Hospital Bauchi, Nigeria \\ ${ }^{2}$ Federal Teaching Hospital Gombe, Nigeria \\ ${ }^{3}$ Federal Medical Center Yola, Adamawa state, Nigeria \\ *Corresponding Author's E-mail: jacobdunga@yahoo.com
}

Abstract

\begin{abstract}
Background: Nigeria suffered waves of Highly Pathogenic Avian Influenza (HPAl) outbreaks that peaked twice in February 2006 and February 2007. The outbreaks affected 3,057 commercial and rural household farms causing 1.3 million of the country's poultry destroyed. The burden of Influenza is likely to be under estimated and because of the unstable nature of the virus it call for the mapping of this virus to specific region, this study therefore seeks to identify the types, prevalence, strain, predilection of the organism so as to monitor the occurrence of influenza in this part of the country with the aim of providing a base line level of intervention of influenza virus and find the candidate virus for vaccine selection and production. This study is a cross sectional survey, our target populations were all pediatric and adult patients admitted at the pediatrics and internal medicine department of ATBUTH who has met the criteria for presence of cough, fever $\left(>37^{\circ} \mathrm{C}\right)$, nasal congestion and dyspnoea. Samples were collected and analyzed using the polymerase chain reaction (PCR), for the presence and sub typing of influenza viruses in respiratory specimens. Overall $5 \%$ of the samples collected tested positive for human influenza type A and B. During average epidemics, overall attack rates are estimated to be $10-20 \%$, but in certain susceptible populations such as schoolchildren or nursing home residents, attack rates of $40-50 \%$ may occur. $60 \%$ of the positive results were among the female samples where as $40 \%$ from the male samples.
\end{abstract}

Keywords: Human influenza virus, Prevalence, Types, Subtypes and Predilections.

\section{INTRODUCTION}

Nigeria suffered waves of Highly Pathogenic Avian Influenza (HPAl) outbreaks that peaked twice in February 2006 and February 2007. The outbreaks affected 3,057 commercial and rural household farms causing 1.3 million of the country's 160 million poultry to be destroyed at the cost of $\$ 5.4 \mathrm{M}$ paid in compensation by the Government of Nigeria (Federal Department of Livestock [FDL], 2008) (Ibrahim et al.,2012), (Scholtissely et al., 1978).

The unstable nature of the virus strain calls for mapping of HPAI to specific state with the aim of utilizing specific regional or state level approach in prevention and management of HPAl.

Influenza virus particularly type A infect large number of warm blooded animals, including wild birds, domestic birds, pigs, horses and humans influenza virus can switch host to form new lineage in novel hosts, the most significant of this is the emergence of antigenic ally novel influenza $A$ in humans leading to pandemics (Jeffery et al., 2010). Pandemics occur when a new avian influenza strain acquires the ability to infect people and to spread 
easily person to person. This can occur in two ways: Reassortment due to exchange of seasonal and avian influenza genes in a person or pig infected with both strains and Mutation, when an avian strain becomes more transmissible through adaptive mutation of the virus during human avian influenza infection (Hay et al., 2011).

Influenza viruses are sub typed according to surface glycoprotein: hemagglutinin (HA) and neuraminidase (NA) Currently, there are 16 hemagglutinin $(\mathrm{H} 1$ to $\mathrm{H} 16)$ and 9 neuraminidases ( $\mathrm{N} 1$ to $\mathrm{N} 9$ ), about 144 possible sub-types have been identified (International committee on Toxonomy of virus 2015).

Hemagglutinin attaches the virus to the surface of the host cell which enables the virus to replicate while Neuraminidase lets the newly replicated viruses out of the cell to infect more cells. The Influenza viruses are divided into three main types: influenza $A, B$, and $C$, the $A$ viruses infect birds and other animals, as well as humans, it is the source of seasonal influenza epidemics and all pandemics while $B$ and $C$ viruses infect humans only and are not associated with pandemics (International committee on Toxonomy of virus 2015). Human influenza originates as avian (birds) influenza, typically from migratory water bird to domestic birds, swine and then human infection occurs as a results of consumption or contact with these domestic animals. (Hay et al.,1416). Human Influenza under goes mutation through "Antigenic Drift and Shift "which enhances its pathogenicity (Hay et al., 1416). Antigenic Drift - Point mutations in HA or NA generally results in relatively small changes in virus, these are small changes in the genes of influenza viruses that happened continually over time as the virus replicates while antigenic Shift is as a result of reassortment of gene segments leading to novel and potentially pandemic strains. Influenza viruses mutate by antigenic drift most times, antigenic shift happens only occasionally (Hay et al., 1416), (Ioannis et al.,2016). Influenza viruses spreads easily from person to person through coughing and sneezing and transmitted by inhaling respiratory aerosols containing the virus, produced when infected person talks, coughs, or sneezes and by touching an infected person or an item contaminated with the virus and then touching the eyes, nose, or mouth (Carat et al., 2008). The incubation period is usually 3 to 7 days depends on the strain of virus, dose of inoculums, age and immune status of bird, management and environmental factors plays a significant role in the infectivity of the virus (Carat et al., 2008). Major clinical presentation include Sudden onset of fever, headache, muscle aches, severe weakness, respiratory symptoms, e.g., cough, sore throat, difficulty breathing (loannis et al.,2016), (Carat et al., 2008).

Pandemics are less predictable, not always in winter, great variations occurs in mortality, severity of illness, and pattern of illness or age most severely affected, rapid surge in number of cases over brief period of time can occur, often measured in weeks and it tend to occur in waves of 6 - 8 weeks, subsequent waves may be more or less severe, more than one wave of influenza is likely, gaps between the waves may be weeks or months, a subsequent wave can be worse than the first (Recommended Composition of Influenza Virus in 2006)

\section{Objective}

The burden of Influenza is likely to be under estimated, this study therefore seeks to identify the types, prevalence, strain and predilection of the organism so as to monitor the occurrence of influenza in this part of the country with the aim of providing a base line level of intervention of influenza virus admitted in this hospital, this will also help give an insight into the candidate virus for vaccine selection and production, similarly it will provide a foundation for detecting outbreaks and pandemics or emergence of a novel strain of influenza so as to create an early warning system to trigger a rapid public health response and advocate for a targeted approach in terms of vaccine procurement and distribution.

\section{METHODS}

This study is a cross sectional survey, our target populations were all pediatric and adult patients admitted at the pediatrics and internal medicine department of ATBUTH who has met the criteria for presence of cough, fever $\left(>37^{\circ} \mathrm{C}\right)$, nasal congestion and dyspnoea. Samples were collected between $8.00 \mathrm{am}$ and $8.00 \mathrm{pm}$. Samples were analyzed using the polymerase chain reaction (PCR) at of national influenza reference laboratory (NIRL) Abuja Nigeria. Samples were analyzed for the presence and sub typing of influenza viruses in respiratory specimens which did not require invitro isolation. Here, primers and probes were used for typing and sub typing. Because the influenza genome consists of singlestranded ribonucleic acids (RNA), complimentary deoxyribonucleic acids (cDNA) were synthesized using the reverse-transcriptase (rt) enzyme prior to the PCR reaction. Therefore any copy of the viral RNA specimens that tested positive for a novel or new subtype of influenza virus were sent to the CDC Atlanta for confirmation specimens were sent with the original sample in case of possible contamination. The results of this technique were obtained within $24 \quad-72 \mathrm{hrs}$. Appropriate controls that identify poor-quality of samples e.g. extraction were utilized to checkmate or avoid false positive results, the most common cause of false-positive 
016 J. Med. Med. Sci.

Table 1. Frequency of Influenza virus based on age group

\begin{tabular}{lccc}
\hline Age group & Frequency- $\mathbf{n}(\%)$ & Positive results/age Grp & Age related Flu types \\
\hline $0-12 \mathrm{mo}$ & $22(22 \%)$ & 0 & \\
$13 \mathrm{mo}-5 \mathrm{y}$ & $29(29 \%)$ & $3(\mathrm{M} 2, \mathrm{~F} 1)$ & Flu A 2, Flu B 1 \\
$6-25 \mathrm{y}$ & $27(27 \%)$ & $1(\mathrm{~F})$ & Flu A 1, Flu B 0 \\
$26-45 \mathrm{y}$ & $13(13 \%)$ & $1(\mathrm{~F})$ & Flu A \\
$46-65 \mathrm{y}$ & $6(6 \%)$ & 0 & \\
$>65 \mathrm{y}$ & $3(3 \%)$ & 0 & \\
\hline
\end{tabular}

Table 2. Frequency of Human Influenza by sex

\begin{tabular}{lccc}
\hline Sex & Frequency & Confirmed cases & Not confirmed \\
\hline Male & 51 & Flu A 1, Flu B 1 & 49 \\
Female & 49 & Flu A 3, Flu B 0 & 46 \\
Total & 60 & Flu A 4, Flu B 1 & 95 \\
\hline
\end{tabular}

results is contamination, this was mitigated by the use of real-time rt-PCR operating in a contained system. Specimens were kept at $-70{ }^{\circ} \mathrm{C}$ and were transporting to the National reference lab in cold chain. Human Influenza positivity was considered as the positive outcome variable where as positive contact with animal or human cases were considered as the exposure variables for this study, possible confounders were presence of other respiratory tract infections e.g Pneumonias, Tuberculosis, respiratory synctytial virus, laryngitis and sinusitis.

Sample size was determined by the number of samples collected for nasal and oral swabs within a period of 12 months.

\section{Data management and analysis}

Results are presented as frequency of Human Influenza virus and its subtypes for both sex and age groups. The result were stored on secured excel data sheet and was analyzed using a statiscal package for social science (SPSS) version 21.

Means $( \pm S D)$ were used to describe continuous variables and proportions were used for categorical data. Twotailed student's t-test was used for comparisons of group means. When comparing groups of subjects, the chisquared $\left(X^{2}\right)$ test was applied to determine the significance of the differences observed.

\section{Ethical approval}

This was obtained from the ethical committee of the ATBUTH where the study was conducted.

\section{RESULTS}

There were 100 samples collected for this study. 49(49\%) of the samples were from female patients, while 51 (51\%) were samples collected from male patient. The age groups are as stated in Table 1 above.

\section{DISCUSSION}

This study is cross section study that was conducted to determine the frequency, types and predilection of human influenza virus among patient admitted with severe acute respiratory infection (SARI) in a tertiary hospital in Nigeria.

Overall $5 \%$ of the samples collected tested positive for human influenza type A and B. During average epidemics, overall attack rates are estimated to be 10$20 \%$, but in certain susceptible populations such as schoolchildren or nursing home residents, attack rates of $40-50 \%$ may occur. (Glezen and Couch 1978) 60\% of the positive result was among the female samples where as 


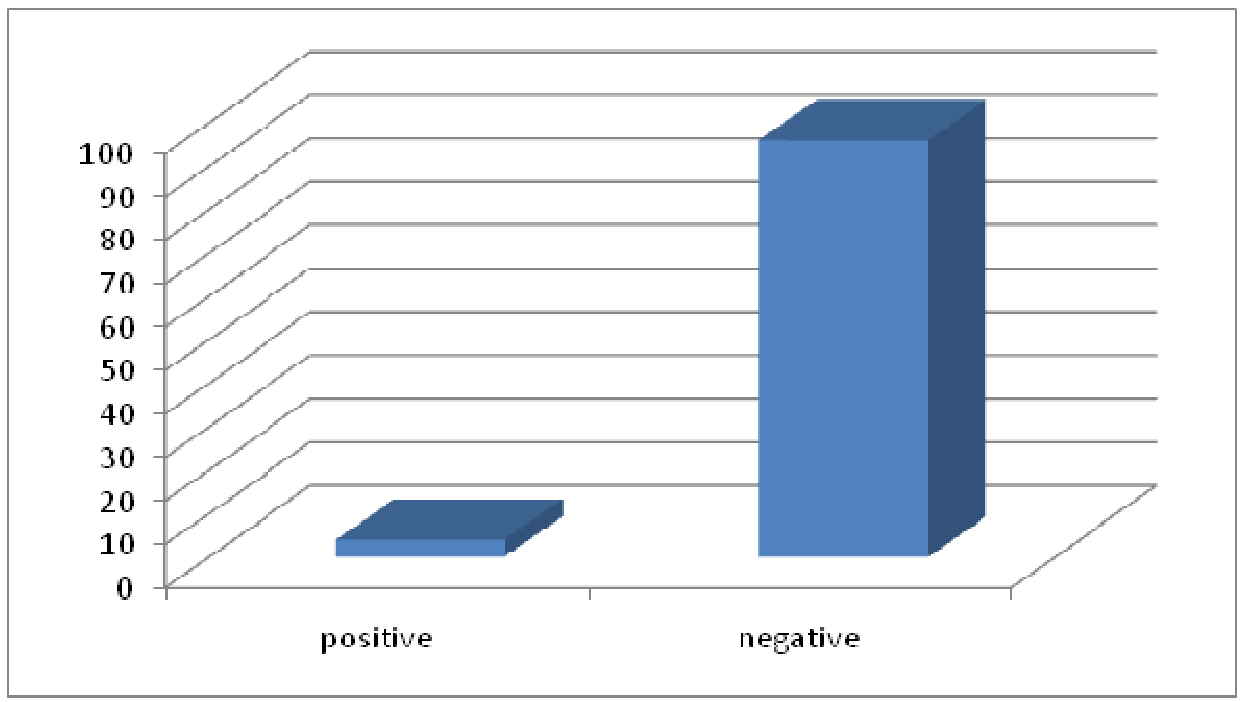

Figure 1. Frequency of Human Influenza \%

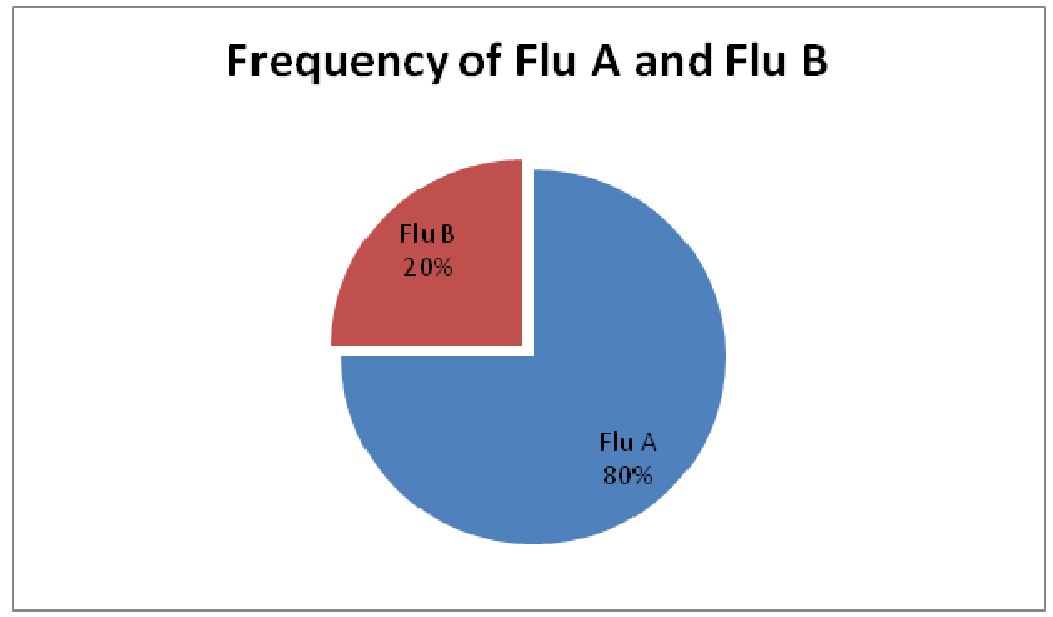

Figure 2: Frequency of Flu A and Flu B among subjects

$40 \%$ from the male samples. Similar study also shows female predilection. (Sharma et al.,2016), even though some studies have shown higher mortality among male children with respiratory tract viral infection including avian influenza. (Poul et al., 2015) Among the positive sample about $80 \%$ were positive for Human Influenza type A (Flu A), where as $20 \%$ where positive for Human Influenza type B (Flu B).

In Nigeria of the 2803 specimens tested, 217 (7.7\%) were positive for influenza viruses, 167 (8\%) were from subjects with influenza like illness (ILI), 17 (5\%) were from subjects with severe acute respiratory illness (SARI) and 33 were from subjects with unclassified condition. During the pre-pandemic period, subtype H3N2 was found to be the dominant circulating influenza $A$ virus subtype while during the 2009 pandemic, influenza A virus subtype $\mathrm{H} 1 \mathrm{~N} 1$ replaced $\mathrm{H} 3 \mathrm{~N} 2$ as the dominant circulating virus. (Ibrahim et al.,2012), (Scholtissely et al., 1978) Among persons with ILI, A (H1N1) pdm09 was most frequently found in children aged 5-17 years, whereas among subjects with SARI, it was most frequently found in persons aged 65 years. The 
percentage of specimens that tested positive for influenza virus peaked at $18.9 \%$ in 2010 and majority were $A$ (H1N1) pdm09 1

In sub-Saharan Africa, only Burkina-Faso, Cameroon, Ethiopia, Kenya, Madagascar, Rwanda and Mauritius reported low influenza activity with influenza A (H3N2) being predominant in DR Congo, Kenya, Mauritius and influenza $A(\mathrm{H} 1 \mathrm{~N} 1)$ pdm09 in Ivory Coast, with influenza $B$ being predominant in Niger, and Uganda. In Ethiopia, Ghana, Tanzania, Madagascar and Togo influenza A and influenza $B$ have been identified in similar proportions in 2012 (WHO Influenza update April 2012).

In tropical Asia, Influenza activity in this region is decreasing or at low or undetectable levels. Although influenza $B$ remains the most commonly detected influenza type in the region, a high proportion of influenza A (H1N1) pdm09 has been detected in India and Bangladesh in the past. In southern China, of the 1,074 specimen that tested positive for influenza. Of the positive specimens, $67 \%$ were influenza A (H3N2), 17\% were influenza A unsubtyped, and only $17 \%$ were influenza $B$, indicating an increase in the proportion of $A$ (H3N2) cases in this region (WHO Influenza update April 2012).

There were more cases of human influenza among the age group 1 - 5years representing $3 \%$ of total samples collected compared to $1 \%$ each for $6-25 y e a r s$ and 26 - 45years, the incidence were found to be less or absent from 45years and above. Studies conducted during both pandemic years and interpandemic periods demonstrate that age-specific attack rates are often highest among schoolchildren. ${ }^{11}$ Family studies conducted in Houston (Taber et al., 1981) and documented age-specific attack rates during various epidemics during the 1970s and 1980s demonstrated high rates of infection in school-age children and the importance of schoolchildren as vehicles of infection within families (Fox et al., 1982).

About $33 \%$ of patient with influenza are asymptomatic (Carat et al.,2008),(Suzuki et al.,2007) Influenza reaches peak prevalence in winter and because of the variability of winter period in northern and southern hemisphere, there are actually 2 flu seasons each year (Dolan et al.,2013). Flu occur seasonally rather than uniformly throughout the year.

One possible explanation is that, because people are indoors more often during the winter, they are in close contact more often, and this promotes transmission from person to person, another factor is that cold temperatures lead to drier air, which may dehydrate mucus, preventing the body from effectively expelling virus particles. The virus also survives longer on surfaces at colder temperatures and aerosol transmission of the virus is highest in cold environments (less than $5^{\circ} \mathrm{C}$ ) with low relative humidity (Lowen et al.,.2007).

\section{CONCLUSION}

The burden of Influenza is likely to be under estimated in this part of the country, Avian influenza infection even though has less prevalence, it is not an uncommon infection in Nigeria and northeastern region, however concerted effort has not been made in the past to identify and type these virus, and determine its predilections, it is worth of note that this study has revealed that avian influenza type $A$ is commoner than $B$ and $C$ and it has much predilection to female and under 5 age group, with seasonality in occurrence. More research is needed to subtype and identify novel types during outbreaks of avian influenza.

\section{ACKNOWLEDGEMENT}

We wish to sincerely thank the National Influenza Survellance Unit and reference laboratory of Nigeria for given us such opportunity to conduct this research, the laboratory unit has help analyze all our samples, also we recognize the effort of the entire team of this research in this hospital who have worked round the clock to produce these peace of article. We also wish to thank the entire management of Abubakar Tafawa Balewa University Teaching Hospital for given us the enabling environment to carry out this research.

\section{REFERENCES}

Carat F, Vergu E, Ferguson NM, Lemaitre M, Cauchemez S, Leach S (2008)Time line of infection and disease in human influenza $A m$ Jour of epid 167 (7): 775-85

Dolan GP, Harris RC Clarkson M, Sokal R, Morgan G (2013). Vaccination of healthcare workers to protect patients at increased risk of acute respiratory disease: summary of a systematic review. Influenza and other respiratory viruses.; (7): 2: 93-6.

Fox JP, Hall CE, Cooney MK (1982). Influenza virus infections in Seattle families, 1975-1979. Methods and the occurrence of infections by time and age.Am. J. Epidemiol. 116:212-27 3

Glezen WP, Couch RB. (1978). Interpandemic influenza in the Houston area, 1974-76. N. Engl. J. Med. 298:587-92 31.

Hay A, Gregory V, Douglas A, Lin Y (1416). The evolution of human influenza viruses" Philos Trans R Soc Lond B Biol Sci. $3561861-$ 70.

Hay AJ, Gre gory V, Douglass AR, Lin YP (2011). The evolution of human Influenza virus. Philos Trans R Soc Lond B Biol Sci 29; 356 (1416):1861-70

Ibrahim TD, Andrew MM, Sonja JO, Inzure H, Aishatu BG (2012). Influenza virus in Nigeria, 2009-2010 result from first 17 month of National Influenza sentinel surveillance. J inft Dis 206 (1): 121-28

International committee on Toxonomy of virus (ICTV) (2015). Virus Toxonomy released 15 June.

loannis S, Xanthoula R, Ben P, Mart CM De Jong (2016). Mutations in the haemagglutinin protein and their effect in transmission of highly pathogenic avian influenza (HPAl) H5N1virus in sub-optimally vaccinated chickens Vaccine, 34 : (46), 4 Nov ;5512-5518.

Jeffery K, Taubenberger MD (2010). David M morens. Influenza the once and future pandemic Pub health Resp; 125 (3):16-26 
Lowen AC, Mubareka S, Steel J, Palese P. Influenza virus transmission is dependent on relative humidity and temperature PLOS Pathogens.2007; 3 (10): e151.

Poul K S, Wilson W S, Tsz C L, Kam LH (2015). Hospitalization Incidence, Mortality, and Seasonality of Common Respiratory Viruses over a Period of 15 Years in a Developed Subtropical City.Med (Baltimore) Nov; 94 (46): e 2024 WHO Influenza update April 2012

Recommended Composition of Influenza Virus in 2006-2007 (2006). Influenza Season WHO report Feb

Scholtissely KC, Rohde W, Von Hoyingin V (1978). Origin of human Influenza Virus subtypes H2N2 and H3N3 Virology;87:13-20
Sharma P, Gupta S, Singh D, Verma S, Kanga A (2016). Influenza A (H1N1) pdmo9 cases in sub- Himalayan region, 2014-2015 India India J pathol Microbial; (59):63-5.

Suzuki E, Ichihara K, Johnson AM (2007). Natural course of fever during influenza virus infection in children. Clin Pediatr (Phila).; 46 (1): 76-9.

Taber LH, Paredes A, Glezen WP (1981). Infection with influenza A/Victoria virus in Houston families. J. Hyg. 86:303-13 34

Yu-Chia H, Tsung-Zu W, Ding-PL, Pei-Lan S, Luan-Yin C, Chun-Yi L, Chin-Yun L, Fu-Yuan H, Li-Min H (2006). Influenza pandemics: past present and future Jour of the Formosan Med Associ; 105) (1): :1-6 